\title{
Analytical Calculation of Air-gap Magnetic Field Distribution in Transverse-Flux Machines
}

\author{
Jaime R. Anglada and Suleiman M. Sharkh \\ Faculty of Engineering and the Environment, University of Southampton, UK \\ Emails: \{j.renedo-anglada, suleiman $\} @$ soton.ac.uk
}

\begin{abstract}
Analytical methods are useful tools in the design of rotating electric machines because the simulation times tend to be low and they provide a good insight. However, in the analysis of non-traditional machines, like the transverse-flux machine (TFM), the most common approach is to use finite element analysis (FEA). In this paper we show how to use the complex permeance (CP) methodology to obtain the magnetic field distribution on the air-gap of a homopolar TFM produced by the stator windings. The properties of the $\mathrm{CP}$ function are based on conformal mapping theory. Also, we propose an algorithm to estimate the parameters of the $C P$ function after evaluating the function in a set of randomly generated number of points, which reduces the computation time significantly. The proposed methodology is applied to a single-sided TFM showing that the results are consistent with FEA calculations.
\end{abstract}

\section{INTRODUCTION}

The magnetic flux-density distribution in the air-gap of an electric machine determines the performance parameters like the torque and the induced electromotive force. For this reason it is essential to have accurate models to describe the electromechanical interaction that takes place in order to design better machines.

Transverse-flux machines (TFM) are very interesting devices because they can achieve high torque densities and therefore they are suitable for direct drive applications [1], [2], [3]; in references [1], [2] this device is called variablereluctance permanent-magnet (VRPM) machine. However, the magnetic field distribution is intrinsically three-dimensional making the task of modelling difficult. For this reason the current trend is to use 3D finite element analysis (FEA) which is time consuming and does not readily provide an insight. Other alternative is to use refined magnetic equivalent circuits, which is a semi-numerical method and is reported to have lower computation times than FEA [4], [5]. Therefore analytical methods remain very useful tools for a quick first design approach.

The complex permeance method proposed by Zarko et al. [7], [8], [9] uses conformal mapping to obtain a complex permeance $(\mathrm{CP})$ function that modulates the magnetic field distribution from the slotless configuration. One of the main disadvantages of the complex permeance method proposed by Zarko is that the computation time is relatively high because of the necessity of evaluating the permeance function in a great number of points to generate whole waveform [10]. This paper presents a different approach to the complex permeance method adapting it for a homopolar field distribution, which is the case of the machine under study. The methodology proposed in this paper to estimate the parameters of the permeance function reduces the computation time significantly because the number of points in which the function has to be evaluated is lower. The significant reduction in the computation time presents an improvement in the context of the design and optimisation of electric machines.

The paper starts with a description of the single-sided TFM under study. Section III describes the application of conformal mapping to obtain the $\mathrm{CP}$ function in the air-gap showing the two conformal transformations involved in the process. In section IV the properties of the CP function are described. Next, in section $\mathrm{V}$ the algorithm to estimate the coefficients of the $\mathrm{CP}$ function using random sampling is presented. Finally, the methodology is applied to the TFM built at the University of Southampton [1], [2] and the results are compared with 2D and 3D FEA for validation.

\section{The Single-Sided TFM Machine Under Study}

Fig. 1 shows the front view and the cross-section of the machine under study, which is an inverted surface-magnet configuration [1], [2]. The stator is two-phase, each phase has a circular coil that links and magnetizes $20 \mathrm{C}$-cores producing a homopolar magnetic field distribution. The radial flux interacts with an array of 40 permanent magnets with alternating polarity. The dimensions of the machine studied are shown in table I.

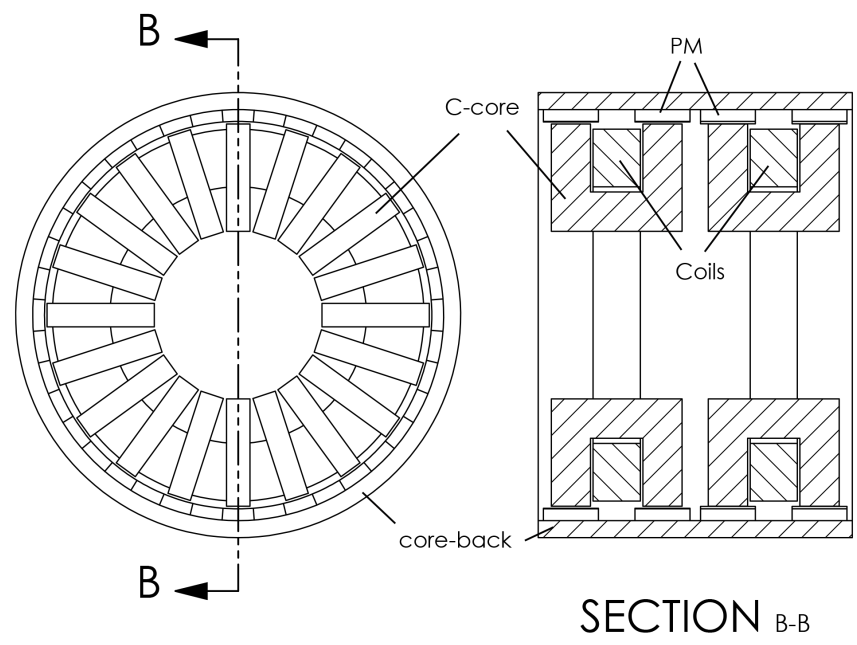

Fig. 1. Front view and cut of a single sided TFM under study. 


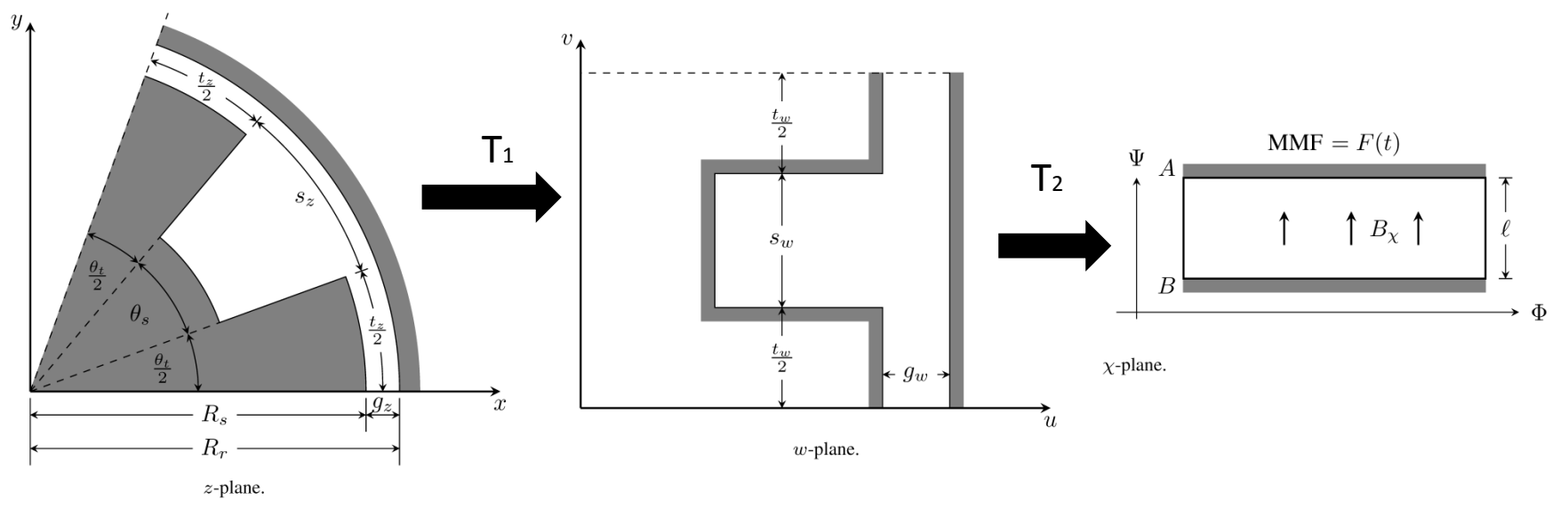

Fig. 2. Conformal transformations required to obtain the magnetic field distribution in the air-gap.

TABLE I

Parameters of THE TF MACHINE

\begin{tabular}{lcc}
\hline Quantity & Symbol & Value \\
\hline Stator radius & $R_{s}$ & $73 \mathrm{~mm}$ \\
Rotor radius & $R_{r}$ & $78.5 \mathrm{~mm}$ \\
Air-gap length & $g_{z}$ & $5.5 \mathrm{~mm}$ \\
Magnet thickness & $d_{m}$ & $4.51 \mathrm{~mm}$ \\
Pole pitch & $\theta_{\lambda}$ & $18^{\circ}$ \\
Tooth pitch & $\theta_{t}$ & $7.02^{\circ}$ \\
Slot pitch & $\theta_{s}$ & $10.98^{\circ}$ \\
Number of C-cores & $n$ & 20 \\
\hline
\end{tabular}

This particular topology has the property of high torque density. However, the path of the magnetic flux is threedimensional and therefore difficult to model analytically.

\section{FiEld SOlution IN THE AIR-GAP}

The air-gap of the machine under study has a toothed member (each C-core can be considered a tooth) and a smooth coreback. Fig. 2 shows the $z$-plane, which is the real geometry, and the transformed domains ( $w$ and $\chi$ planes), which are described later in the paper. The magnetic field distribution is obtained using a complex permeance (CP) function [7], [8], [9] adapted for a homopolar field distribution. We are assuming that the permeability of the iron is infinite and the effect of saturation is negligible.

We present an alternative interpretation of the CP function in order to simplify the final expression of the magnetic field distribution. The function $\lambda(\theta, r)$ modulates the scalar value of $B_{s}(t)$ that is the instantaneous magnetic flux-density produced by the stator windings in the simplified rectangular slotless geometry defined as follows:

$$
B_{s}(t)=\frac{\mu_{0} F(t)}{g_{z}},
$$

where $F(t)$ is the instantaneous magneto-motive force (MMF) produced by the stator windings and $g$ is the effective air-gap length. The function $\lambda(\theta, r)$ depends only on the geometric properties of the air-gap that give the shape. The scalar value of $B_{s}(t)$ gives the magnitude to the function of the magnetic field distribution $\vec{B}(t, \theta, r)$.

The function $\lambda(\theta, r)$ is calculated in such a way that the real part corresponds to the radial component and the imaginary to the tangential one. In this paper the variable $\theta$ is expressed in electrical radians, such that a pole pitch is $2 \pi$; to change to mechanical radians it is necessary to divide by the number of pairs of poles. Accordingly, the expression of the magnetic field distribution in the air-gap expressed as a vector is:

$$
\vec{B}(t, \theta, r)=B_{s}(t)\left[\operatorname{Re}\{\lambda(\theta, r)\} \vec{u}_{r}+\operatorname{Im}\{\lambda(\theta, r)\} \vec{u}_{\theta}\right] .
$$

The value of $\lambda(\theta, r)$ is obtained using conformal mapping theory by transforming the original domain ( $z$-plane) into a new one in which we know the solution, in this case the new domain is a rectangle ( $\chi$-plane) where the magnetic field is constant [11]. To achieve this there are two conformal transformations to be done, a proportional-logarithmic transformation [12] that transforms the circular geometry into a rectangular developed model and a Schwarz-Christoffel transformation that maps the developed model into a rectangle [11], [13], [7], [8], [9].

\section{A. Proportional-Logarithmic Transformation $\left(T_{1}\right)$}

The first conformal transformation ( $T_{1}$ in Fig. 2$)$ maps the circular geometry of the $z$-plane into a rectangular geometry in the $w$-plane. To achieve this $T_{1}$ has to be a proportionallogarithmic transformation [12]. The proportionality constant $R_{g}$ chosen is the radius of the middle of the air-gap in this case. The transformation to obtain the $w$-plane is the following:

$$
w=R_{g} \log (z) .
$$

According to the theory of conformal mapping [11], [13], [14] the relationship between the magnetic field in $z$ and $w$ planes expressed as complex numbers:

$$
B_{z}=B_{w}\left(\frac{d w}{d z}\right)^{*}=B_{w}\left(\frac{R_{g}}{r_{z}} e^{j \theta_{z}}\right)
$$


The term that multiplies $B_{w}$ in (3) has a term that is a scale factor $\left(\frac{R_{g}}{r}\right)$ and a second term $\left(e^{j \theta_{z}}\right)$ that transforms the real component into radial and the imaginary one into tangential. To obtain the magnetic field at a particular point of the $z$ plane it is sufficient to know the value of the field on the $w$-plane and the derivative of the transformation evaluated in that point. If we define the relative permeance associated to the proportional-logarithmic transformation as

$$
\lambda_{\log }(r)=\frac{R_{g}}{r}
$$

then, the expression of the magnetic field in the $z$-plane as a vector $\vec{B}_{z}$ in radial and tangential components is as follows

$$
\vec{B}_{z}=\left[\operatorname{Re}\left\{B_{w}\right\} \vec{u}_{r}+\operatorname{Im}\left\{B_{w}\right\} \vec{u}_{\theta}\right] \lambda_{\log }(r) .
$$

\section{B. Schwarz-Christoffel Transformation $\left(T_{2}\right)$}

The details of Schwarz-Christoffel transformations and their application to solve this problem are given in [11], [13], [14]. According to the literature this transformation is sometimes called Numerical Schwarz-Christoffel Transformation because the equation of the transformation is not explicit, in this paper the solution is obtained with the SC Toolbox developed by Driscoll [15].

The starting point is the polygon in the $w$-plane that needs to be transformed into a rectangle that is the $\chi$-plane, shown schematically in Fig. 2, in which the magnetic field distribution is known. The general equation of the SC transformation is as follows [14]:

$$
w=f(\chi)=K_{1} \int \prod_{k=1}^{N}\left(\chi-\chi_{k}\right)^{\frac{\alpha_{k}}{\pi}-1} d \chi+K_{2},
$$

where $K_{1}$ and $K_{2}$ are integration constants and $\alpha_{k}$ are the interior angles of the polygon. The function $\hat{f}(w)$ is defined as the inverse of $f(\chi)$ :

$$
\chi=\hat{f}(w) .
$$

Even though there is not an analytical expression of $\hat{f}(w)$ the derivative of the transformation of the $w$-plane into the $\chi$-plane is defined as follows (taking into account that $\chi$ is a function of $w$ )

$$
\frac{d \chi}{d w}=\hat{f}^{\prime}(w)
$$

The relationship between the magnetic fields is given by

$$
B_{w}=B_{\chi}\left(\frac{d \chi}{d w}\right)^{*}=B_{\chi}\left\{\hat{f}^{\prime}(w)\right\}^{*} .
$$

The functions $\hat{f}(w)$ and $\hat{f}^{\prime}(w)$ can be evaluated in each point with the SC Toolbox once the map is created.

The SC transformation is such that the domain in the $\chi$ plane is the rectangle shown in Fig. 2. The solution to the Laplace equation considering an MMF $F(t)$ is the following:

$$
B_{\chi}(t)=\frac{\mu_{0} F(t)}{\ell}
$$

where the length $\ell$ can be calculated as the following:

$$
\ell=\left|\chi_{A}-\chi_{B}\right|
$$

The SC Toolbox gives the option of obtaining $\chi_{i}=\hat{f}\left(w_{i}\right)$ making the calculation of $\ell$ trivial because is just the length of the side of the rectangle on the canonical domain. The field in the $w$-plane can be expressed as

$$
B_{w}=B_{\chi}\left\{\hat{f}^{\prime}(w)\right\}^{*}=B_{s}(t) \frac{g_{z}}{\ell}\left\{\hat{f}^{\prime}(w)\right\}^{*},
$$

the term that multiplies $B_{s}(t)$ is the relative complex permeance associated with the SC transformation taking into account that $w$ is a function of $\theta$ and $r$ :

$$
\lambda_{S C}(\theta, r)=\frac{g_{z}}{\ell}\left\{\hat{f}^{\prime}(w(\theta, r))\right\}^{*} .
$$

For the polygon considered here there is not an explicit expression $\hat{f}$ but with the SC Toolbox [15] it can be evaluated at any point and also the derivative can be evaluated. Consequently, the function $\lambda_{S C}(\theta, r)$ cannot be obtained directly but can be evaluated at every point of the domain.

\section{PRoperties OF THE CP FunCtion}

The function $\lambda_{\log }(r)$ associated to the logarithmic transformation is a real number and the function $\lambda_{S C}(\theta, r)$ associated to the SC transformation can be separated into real and imaginary part that correspond to the radial and tangential components respectively. The permeance function can be expressed as the product of these two functions as follows:

$$
\lambda(\theta, r)=\lambda_{\log }(r)\left[\lambda_{r}(\theta, r)+j \lambda_{\theta}(\theta, r)\right],
$$

where

$$
\lambda_{S C}(\theta, r)=\lambda_{r}(\theta, r)+j \lambda_{\theta}(\theta, r)
$$

The function $\lambda_{S C}(\theta, r)$ has the same shape as the magnetic field distribution of the rectangular geometry ( $w$-plane in this paper, Fig. 2) therefore for each $r$ the real and imaginary parts can be expressed as Fourier series according to [13] because of the symmetry of the boundary conditions:

$$
\begin{gathered}
\lambda_{r}(\theta, r)=\bar{\lambda}_{r}\left[1+\sum_{n=1}^{\infty} \gamma_{n}(r) \cos (n \theta)\right], \\
\lambda_{\theta}(\theta, r)=\sum_{n=1}^{\infty} \lambda_{\theta n}(r) \sin (n \theta),
\end{gathered}
$$

with the coefficients calculated accordingly:

$$
\begin{gathered}
\bar{\lambda}_{r}=\frac{1}{\pi} \int_{0}^{\pi} \operatorname{Re}\left\{\lambda_{S C}(\theta, r)\right\} d \theta \\
\gamma_{n}(r)=\frac{2}{\pi \bar{\lambda}_{r}} \int_{0}^{\pi} \operatorname{Re}\left\{\lambda_{S C}(\theta, r)\right\} \cos (n \theta) d \theta \\
\lambda_{\theta n}(r)=\frac{2}{\pi} \int_{0}^{\pi} \operatorname{Im}\left\{\lambda_{S C}(\theta, r)\right\} \sin (n \theta) d \theta .
\end{gathered}
$$




$$
[\mathbf{X}]_{K \times N_{c}}=\left[\begin{array}{cccccccccc}
1 & \cos \left(\theta_{1}\right) & \delta_{1} \cos \left(\theta_{1}\right) & \ldots & \delta_{1}^{N_{p}} \cos \left(\theta_{1}\right) & \ldots & \cos \left(N_{h} \theta_{1}\right) & \delta_{1} \cos \left(N_{h} \theta_{1}\right) & \ldots & \delta_{1}^{N_{p}} \cos \left(N_{h} \theta_{1}\right) \\
1 & \cos \left(\theta_{2}\right) & \delta_{2} \cos \left(\theta_{2}\right) & \ldots & \delta_{2}^{N_{p}} \cos \left(\theta_{2}\right) & \ldots & \cos \left(N_{h} \theta_{2}\right) & \delta_{2} \cos \left(N_{h} \theta_{2}\right) & \ldots & \delta_{2}^{N_{p}} \cos \left(N_{h} \theta_{2}\right) \\
\vdots & \vdots & \vdots & \ddots & \vdots & \ddots & \vdots & \vdots & \ddots & \vdots \\
1 & \cos \left(\theta_{K}\right) & \delta_{K} \cos \left(\theta_{K}\right) & \ldots & \delta_{K}^{N_{p}} \cos \left(\theta_{K}\right) & \ldots & \cos \left(N_{h} \theta_{K}\right) & \delta_{K} \cos \left(N_{h} \theta_{K}\right) & \ldots & \delta_{K}^{N_{p}} \cos \left(N_{h} \theta_{K}\right)
\end{array}\right]
$$

The total flux crossing the air-gap has to be constant and therefore $\bar{\lambda}_{r}$ is constant and does not depend on $r$. On the other hand the functions $\gamma_{n}(r)$ and $\lambda_{\theta n}(r)$ have an unknown shape but they can be approximated as polynomials. For the polynomial approximation, instead of using the variable $r$ the distance to the coreback $\delta$ is used:

$$
\delta=R_{g}+\frac{g}{2}-r .
$$

The functions of the Fourier coefficients expressed as polynomials of $\delta$ are as follows

$$
\begin{gathered}
\gamma_{n}(\delta)=\gamma_{n}^{0}+\gamma_{n}^{1} \delta+\gamma_{n}^{2} \delta^{2}+\cdots, \\
\lambda_{\theta n}(\delta)=\lambda_{\theta n}^{1} \delta+\lambda_{\theta n}^{2} \delta^{2}+\lambda_{\theta n}^{3} \delta^{3}+\cdots,
\end{gathered}
$$

where $\gamma_{n}^{0}, \gamma_{n}^{1}, \gamma_{n}^{2}, \ldots$, are the Taylor coefficients for the radial component and $\lambda_{\theta n}^{1}, \lambda_{\theta n}^{2}, \lambda_{\theta n}^{3}, \ldots$, the corresponding ones for the tangential component ${ }^{1}$.

\section{Estimation of the Coefficients of the CP FUNCTION}

One of the main disadvantages of the $\mathrm{CP}$ method implemented in [7], [8], [9] is that the computation time is high because the $\mathrm{CP}$ function needs to be evaluated at each point to obtain the waveform [10]. However, with the proposed methodology for an arbitrary geometry the machine designer can decide the harmonic order, $N_{h}$, and the order of the polynomial of the Taylor series, $N_{p}$, and after that estimate the number of points that have to be evaluated in order to obtain the coefficients. For the radial component of there are $N_{c}$ coefficients $\left(\bar{\lambda}_{r}, \gamma_{n}^{0}, \gamma_{n}^{1}, \gamma_{n}^{2}, \ldots\right)$ and for the tangential component of there are $N_{c \theta}<N_{c}$ coefficients $\left(\lambda_{\theta n}^{1}, \lambda_{\theta n}^{2}, \lambda_{\theta n}^{3}\right.$, ...). Under these conditions the model can be fully defined by evaluating at least $N_{c}$ independent points. If $N_{h}$ is the higher order harmonic and $N_{p}$ the power of the last term of the Fourier series, $N_{c}$ is calculated as follows:

$$
N_{c}=N_{h}\left(N_{p}+1\right)+1 .
$$

To describe the following algorithm to identify the parameters of the permeance function instead of directly calculating

\footnotetext{
${ }^{1}$ The tangential component does not have the $\lambda_{\theta n}^{0}$ term because there is not tangential component at $\delta=0$ in this geometry.
}

$\gamma_{n}(\delta)$ we will first calculate $a_{n}(\delta)$ that is the harmonic amplitude before normalisation:

$$
a_{n}(\delta)=\bar{\lambda}_{r} \gamma_{n}(\delta)
$$

For an arbitrary point $\left(\theta_{i}, r_{i}\right)$ the value of the permeance function:

$$
\operatorname{Re}\left\{\lambda_{S C}\left(\theta_{i}, r_{i}\right)\right\}=\bar{\lambda}_{r}+\sum_{n=1}^{N_{h}} \sum_{m=0}^{N_{p}} a_{n}^{m} \delta_{i}^{m} \cos \left(n \theta_{i}\right),
$$

where $\lambda_{S C}\left(\theta_{i}, r_{i}\right)$ is calculated as follows:

$$
\lambda_{S C}\left(\theta_{i}, r_{i}\right)=\lambda\left(\theta_{i}, r_{i}\right) \frac{r_{i}}{R_{g}} .
$$

If we consider $K$ independent points, with $K>N_{c}$, we have an over determined linear system with $K$ equations and $N_{c}$ unknowns that are the coefficients. (26) can be written in a matrix form as follows:

$$
\mathrm{XC}=\Lambda,
$$

where $[\mathbf{X}]_{K \times N_{c}}$ is the matrix with the points in which the permeance function is evaluated, organised to be consistent with (26); the full matrix is shown at the top of the page. C is the matrix with the coefficients that we want to estimate:

$$
[\mathbf{C}]_{N_{c} \times 1}=\left[\begin{array}{c}
\bar{\lambda}_{r} \\
a_{1}^{0} \\
a_{1}^{1} \\
\vdots \\
a_{1}^{N_{p}} \\
a_{2}^{0} \\
\vdots \\
a_{N_{h}}^{N_{p}}
\end{array}\right] .
$$

$\Lambda$ is the solution vector

$$
[\boldsymbol{\Lambda}]_{K \times 1}=\left[\begin{array}{c}
\operatorname{Re}\left\{\lambda_{S C}\left(\theta_{1}, r_{1}\right)\right\} \\
\operatorname{Re}\left\{\lambda_{S C}\left(\theta_{2}, r_{2}\right)\right\} \\
\vdots \\
\operatorname{Re}\left\{\lambda_{S C}\left(\theta_{K}, r_{K}\right)\right\}
\end{array}\right] .
$$

The coefficients can be estimated using the following linear least squares algorithm:

$$
\mathbf{C}=\left[\left(\mathbf{X}^{t} \mathbf{X}\right)^{-1} \mathbf{X}^{t}\right] \boldsymbol{\Lambda},
$$


where $t$ denotes the transposed matrix.

Once the vector $\mathbf{C}$ is known the real part of the complex permeance function is fully defined. The procedure for the imaginary part is completely analogous. Once the real and the imaginary part are obtained the magnetic field distribution is known as a vector in all the air-gap.

\section{A. Random Sampling}

To improve the reliability of this algorithm we want to make sure that we avoid having any problem in inverting the the matrix $\left[\mathbf{X}^{t} \mathbf{X}\right]$, or what is the same: that we have at least $N_{c}$ independent points. To achieve this we propose to generate a set of random points. It was observed that if we have a given number of points distributed uniformly as a grid and we are trying to detect a high frequency space harmonic whose period is of a similar order of magnitude as the separation of the points; then the matrix $\left[\mathbf{X}^{t} \mathbf{X}\right]$ may produce problems in the inversion. Because of the nature of random sampling this problem does not appear because the points are not equally spaced. Each point is calculated as follows:

$$
\begin{aligned}
\delta_{i} & =d_{m} \cdot \mathbf{R}(0,1), \\
\theta_{i} & =2 \pi \cdot \mathbf{R}(0,1),
\end{aligned}
$$

where $\mathbf{R}(0,1)$ denotes a random variable between 0 and 1 with constant probability density function.

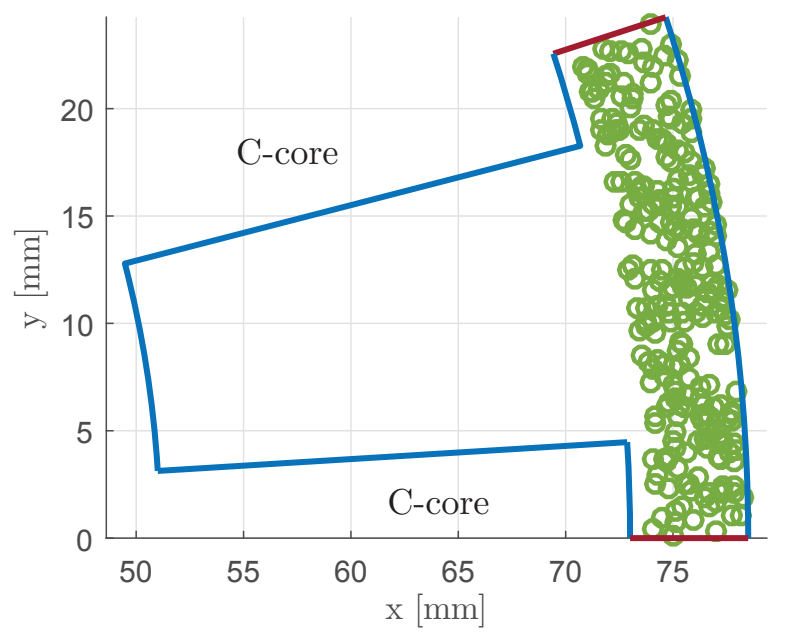

Fig. 3. The set of random points, $S$. In this case it consists 250 random points..

Fig. 3 shows the set of points $S$, which consists in 250 points generated randomly. The improvement in the stability of the algorithm is due to the fact that the points are not equally spaced and therefore the high frequency harmonics can be detected.

\section{RESULTS}

This section illustrates the proposed methodology by its application to the transverse-flux (or VRPM) machine described in section II. This machine was built at the University of
Southampton and has been studied in detail with FEA [1], [2] making it suitable for validation of the proposed methodology.

The windings of the machine have $N_{t}=230$ turns in the configuration analysed in this paper and the rated current $I$ is $10 \mathrm{~A}$. The MMF in each C-core head is calculated as follows

$$
F=\frac{1}{2} N_{t} I=1150 \mathrm{~A}
$$

therefore the magnetic field of the slotless configuration is

$$
B_{s}=\frac{\mu_{0} F}{g}=\frac{4 \pi \cdot 10^{-7} \cdot 1150}{4.5 \cdot 10^{-3}} \approx 0.263 \mathrm{~T} .
$$

If the current was a sinusoidal the value of $B_{s}$ would not be constant but a sinusoidal function as well, the analysis here considering a constant current is the case of a square waveform.

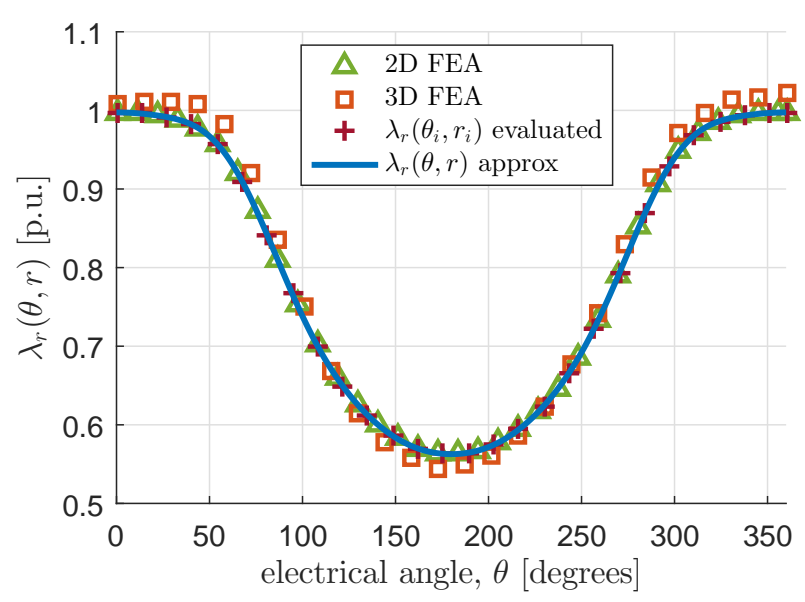

Fig. 4. $\lambda_{r}(\theta, r)$ in the middle of the air-gap, $r=R_{g}$.

Fig. 4 shows the real part of the permeance function at the middle of the air-gap $\left(r=R_{g}\right)$. The green triangles were obtained using 2D FEA with a scalar potential formulation, the orange squares were obtained using 3D magnetostatic FEA, the red crosses are the value of $\lambda_{r}\left(\theta_{i}, r_{i}\right)$ evaluated using the SC Toolbox and the blue line is the approximated model considering 500 random points, the harmonic order $N_{h}=11$ and the polynomial order $N_{p}=5$. The value of $\bar{\lambda}_{r}$ obtained by evaluating the function, 2D FEA and the proposed model has a less than $0.01 \%$ error in this case.

To study the accuracy of the method in the entire domain and not only in the air-gap Fig. 5 shows the harmonic amplitude of the first four harmonics obtained by evaluating the permeance function, 2D FEA and the proposed method. The coefficients of the Fourier series for the comparison were calculated by evaluating (17) and (18) after obtaining the waveform with FEA or by evaluating points with the SC Toolbox. 

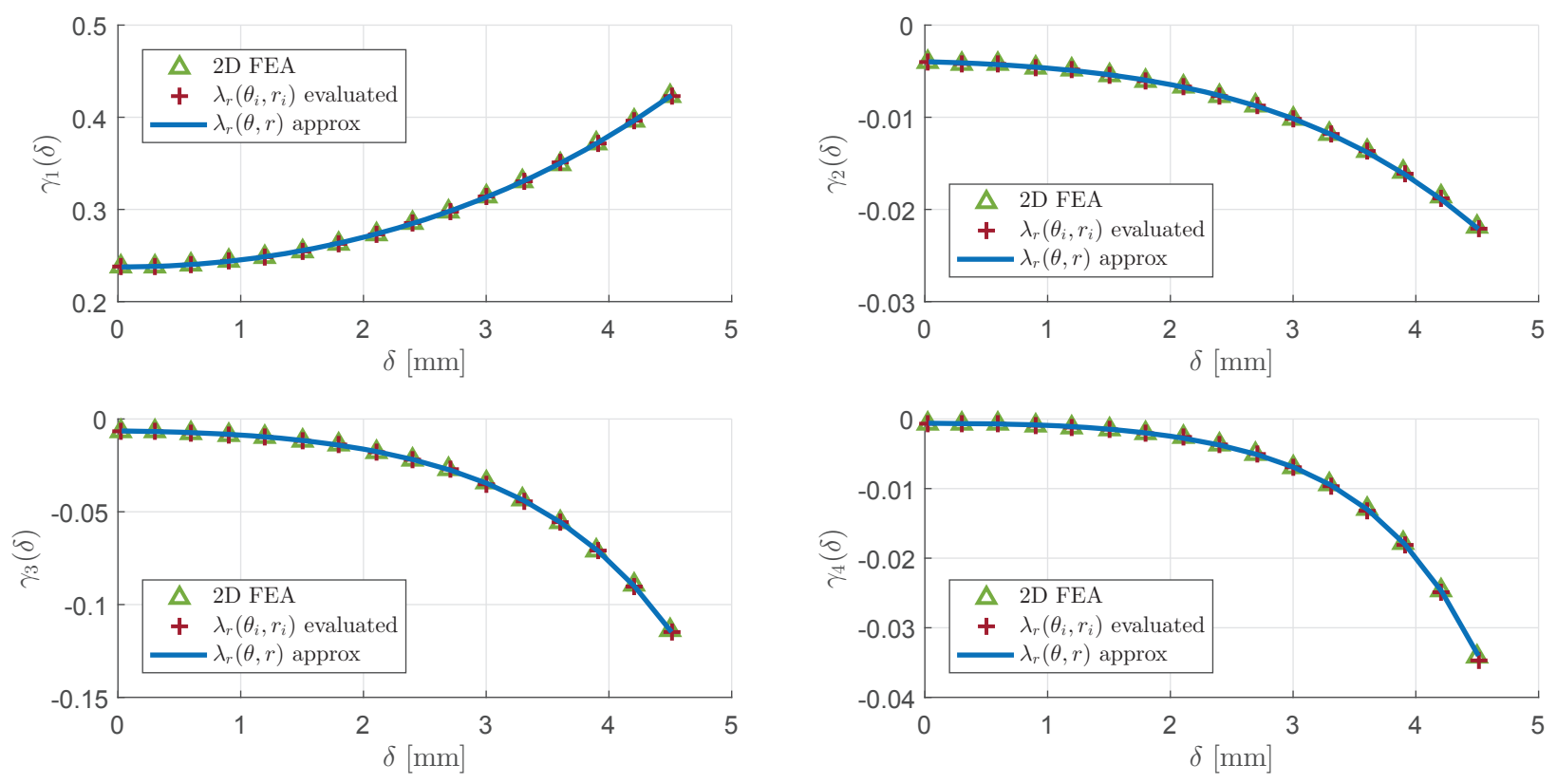

Fig. 5. The amplitude of the first four harmonics of the radial component of the magnetic field $N_{h}=11$ and $N_{p}=5$, with a set of 500 random points.

\section{CONCLUSION}

The paper illustrates how to obtain the magnetic field distribution in the air-gap of electric machines using the CP method combined with random sampling. The case study is a TFM but it can be applied to other machines that have a slotted topology. The results obtained with the methodology presented here are consistent with FEA and with the traditional CP method.

Random sampling can effectively improve the computation time by reducing the number of points at which the $\mathrm{CP}$ function has to be evaluated. To generate the whole waveform of the $\mathrm{CP}$ function at a particular radius, $r$, it is necessary to evaluate a large number of points depending on the accuracy required. With a small set of randomly generated points we can accurately estimate the CP function coefficients in all the air-gap. Random sampling was preferred to uniform sampling because of the improvement of the stability of the algorithm.

The formulation of the problem is such that the shape of the $\mathrm{CP}$ function is deduced from conformal mapping theory and we only have to calculate the coefficients of the polynomials. This allows us to directly estimate the amplitude of the harmonics, which facilitates subsequent analysis of performance.

\section{REFERENCES}

[1] M. Harris and B. Mecrow, "Variable reluctance permanent magnet motors for high specific output," in Electrical Machines and Drives, 1993. Sixth International Conference on (Conf. Publ. No. 376), pp. 437442, Sep 1993.

[2] M. Harris, G. Pajooman, and S. Abu Sharkh, "Performance and design optimisation of electric motors with heteropolar surface magnets and homopolar windings," Electric Power Applications, IEE Proceedings -, vol. 143, pp. 429-436, Nov 1996.
[3] O. Keysan, M. Mueller, A. McDonald, N. Hodgins, and J. Shek, "Designing the c-gen lightweight direct drive generator for wave and tidal energy," Renewable Power Generation, IET, vol. 6, pp. 161-170, May 2012.

[4] V. Ostovic, "A simplified approach to magnetic equivalent-circuit modeling of induction machines," IEEE Transactions on Industry Applications, vol. 24, pp. 308-316, Mar 1988.

[5] M. Amrhein and P. T. Krein, "3-d magnetic equivalent circuit framework for modeling electromechanical devices," IEEE Transactions on Energy Conversion, vol. 24, pp. 397-405, June 2009.

[6] M. Amrhein and P. T. Krein, "Induction machine modeling approach based on 3-d magnetic equivalent circuit framework," IEEE Transactions on Energy Conversion, vol. 25, pp. 339-347, June 2010.

[7] D. Zarko, D. Ban, and T. Lipo, "Analytical calculation of magnetic field distribution in the slotted air gap of a surface permanent-magnet motor using complex relative air-gap permeance," Magnetics, IEEE Transactions on, vol. 42, pp. 1828-1837, July 2006.

[8] K. Boughrara, B. Chikouche, R. Ibtiouen, D. Zarko, and O. Touhami, "Analytical model of slotted air-gap surface mounted permanent-magnet synchronous motor with magnet bars magnetized in the shifting direction," Magnetics, IEEE Transactions on, vol. 45, pp. 747-758, Feb 2009.

[9] K. Boughrara, D. Zarko, R. Ibtiouen, O. Touhami, and A. Rezzoug, "Magnetic field analysis of inset and surface-mounted permanent-magnet synchronous motors using schwarz-christoffel transformation," Magnetics, IEEE Transactions on, vol. 45, pp. 3166-3178, Aug 2009.

[10] Z. Zhu, L. Wu, and Z. Xia, "An accurate subdomain model for magnetic field computation in slotted surface-mounted permanent-magnet machines," Magnetics, IEEE Transactions on, vol. 46, pp. 1100-1115, April 2010.

[11] W. J. Gibbs, Conformal Transformations in Electrical Engineering. Chapman and Hall, 1958.

[12] R. Rabinovici, "Magnetic field analysis of permanent magnet motors," IEEE Transactions on Magnetics, vol. 32, no. 1, pp. 265-269, 1996.

[13] E. Freeman, "The calculation of harmonics, due to slotting, in the fluxdensity waveform of a dynamo-electric machine," Proceedings of the IEE - Part C: Monographs, vol. 109, pp. 581-588, September 1962.

[14] T. A. Driscoll and L. N. Trefethen, Schwarz-Christoffel Mapping. Cambridge ; New York : Cambridge University Press, 2002., 2002.

[15] T. A. Driscoll, Schwarz-Christoffel Toolbox User's Guide: Version 2.3. Newark, DE: Dept. Math. Sci., Univ. Delaware, 2005. 